\title{
Cyclical Patterns Affect Microbial Dynamics in the Water Basin of a Nuclear Research Reactor
}

\author{
Valérie Van Eesbeeck ${ }^{1,2}$, Ruben Props ${ }^{1,3}$, Mohamed Mysara1, Pauline C. M. Petit, \\ Corinne Rivasseau 4 , Jean Armengaud ${ }^{5}$, Pieter Monsieurs' 6 , Jacques Mahillon ${ }^{2}$ and \\ Natalie Leys ${ }^{1 *}$
}

\begin{abstract}
'Microbiology Unit, Environment, Health and Safety Department, Belgian Nuclear Research Centre (SCK CEN), Mol, Belgium, ${ }^{2}$ Food and Environmental Microbiology Laboratory, Earth and Life Institute, Catholic University of Louvain, Louvain-la-Neuve, Belgium, ${ }^{3}$ Center for Microbial Ecology and Technology, Ghent University, Ghent, Belgium, ${ }^{4}$ Institute for Integrative Biology of the Cell (I2BC), CEA, CNRS, Université Paris-Saclay, Paris, France, ${ }^{5}$ Technological Innovations for Detection and Diagnosis Laboratory, CEA, Bagnols-sur-Cèze, France, ${ }^{6}$ Protozoology Research Group, Department of Biomedical Sciences, Institute of Tropical Medicine (ITG), Antwerp, Belgium
\end{abstract}

The BR2 nuclear research reactor in Mol, Belgium, runs in successive phases of operation (cycles) and shutdown, whereby a water basin surrounding the reactor vessel undergoes periodic changes in physico-chemical parameters such as flow rate, temperature, and radiation. The aim of this study was to explore the microbial community in this unique environment and to investigate its long-term dynamics using a $16 \mathrm{~S}$ rRNA amplicon sequencing approach. Results from two sampling campaigns spanning several months showed a clear shift in community profiles: cycles were mostly dominated by two Operational Taxonomic Units (OTUs) assigned to unclassified Gammaproteobacterium and Pelomonas, whereas shutdowns were dominated by an OTU assigned to Methylobacterium. Although 1 year apart, both campaigns showed similar results, indicating that the system remained stable over this 2-year period. The community shifts were linked with changes in physico-chemical parameters by Nonmetric Multidimensional Scaling (NMDS) and correlation analyses. In addition, radiation was hypothesized to cause a decrease in cell number, whereas temperature had the opposite effect. Chemoautotrophic use of $\mathrm{H}_{2}$ and dead cell recycling are proposed to be used as a strategies for nutrient retrieval in this extremely oligotrophic environment.

Keywords: nuclear reactor, ultrapure water, ionizing radiation, 16S rRNA amplicon sequencing, aquatic microbiome, extreme environment

\section{INTRODUCTION}

Waters of nuclear facilities are constantly filtered and deionized to remove dissolved radionuclides, as well as impurities and ions that could become activated. They are also exposed to varying levels of ionizing radiation depending on their proximity with radioactive sources (up to $40 \mathrm{kGy} \mathrm{h}^{-1}$ as estimated in the primary circuit of the BR2 reactor during operation). These ultrapure waters thus represent a rather inhospitable environment for microorganisms. Yet, bacteria, fungi, and microalgae have previously been detected in cooling waters of such facilities, namely in Spent Nuclear Fuel Pools (SNFPs), where spent nuclear fuel is stored in racks under water in order to cool down before being disposed (Santo Domingo et al., 1998; Chicote et al., 2005; Masurat et al., 2005; 
Rivasseau et al., 2016). Microbes can be present in the form of planktonic populations or biofilms, which can adhere to metal surfaces in SNFPs, potentially leading to microbiologically influenced corrosion (MIC; Zhang et al., 1999; Giacobone et al., 2011; Smart et al., 2014). Although this phenomenon has been investigated, together with the radionuclide bioaccumulation potential of some bacterial strains (Jolley, 2002; Tisakova et al., 2013), these studies mainly focused on cultivation-based approaches at a single point in time to identify and characterize the detected microorganisms. However, only a small percentage of environmental bacteria can be cultivated under laboratory conditions (Whitman et al., 1998). This fundamentally limits the potential to investigate the dynamics of the community as a whole. Conversely, with the advent of the next-generation sequencing (NGS) platform and -omics techniques, it has become possible to analyze the DNA from all the members of a microbial population, hereby gaining insights into the entire community. As such, those techniques are starting to get implemented in the study of microbial communities within SNFPs and other watery environments in nuclear reactors (Bagwell et al., 2018; MeGraw et al., 2018; Foster et al., 2020; Petit et al., 2020; Ruiz-Lopez et al., 2020). Nevertheless, knowledge on the evolution of these communities over time is currently lacking.

Instead of the previously described SNFP microbiota (Santo Domingo et al., 1998; Zhang et al., 1999; Chicote et al., 2005; Masurat et al., 2005; Giacobone et al., 2011; Tisakova et al., 2013; Smart et al., 2014; Rivasseau et al., 2016; Bagwell et al., 2018; MeGraw et al., 2018; Foster et al., 2020; Ruiz-Lopez et al., 2020), this work focuses on a similar but more extreme (higher radioactivity) environment consisting of a water basin directly surrounding the vessel of a nuclear reactor. By using a $16 \mathrm{~S}$ rRNA amplicon sequencing approach, it was possible to identify bacterial taxa that went undetected before. Only a single study investigated a similar environment using an equivalent approach, namely the cooling pool of a nuclear reactor in France (Petit et al., 2020), highlighting the novelty of this research. Moreover, instead of sampling a single time point, our sampling campaign covering several months included a time series spanning both active and inactive periods of reactor operation, allowing us to investigate the dynamics of the microbial population over time. As the reactor is running in different phases, where an active period (cycle) is interspersed with periods of shutdown for reactor maintenance, these data shed a new light on the microbial dynamics within this unique environment. Since the transition between cycle and shutdown phases is accompanied by a shift in physical parameters such as temperature and radiation, this sudden change in external conditions was hypothesized to influence the community composition and cell density.

\section{MATERIALS AND METHODS}

\section{Sampling Site and Sample Collection}

The study was conducted at the BR2 nuclear reactor at the Belgian Nuclear Research Centre (SCK CEN) in Mol, Belgium. The sampling environment consisted of an open basin surrounding the reactor vessel (Supplementary Figure 1).
The reactor successively goes through cycles of operation (e.g., production of radioisotopes for medical and industrial use) and shutdown of approximately 30 days each, which are associated with changes in physico-chemical parameters of the water such as flow rate, temperature, and radioactivity. For this study, two sampling campaigns were performed: in the first one, the basin water was studied for three consecutive cycles interspersed with shutdown periods over a period of 8 months (from September 06, 2016 to April 28, 2017). The second one was conducted 1 year later (from April 16, 2018 to June 11, 2018) where it was only monitored for one cycle. In this case, the cell density was also assessed for each sample.

The study of the reactor basin environment represented a challenge in terms of its low bacterial load combined with the presence of radioactivity. For sample collection, a filtration system was therefore designed to minimize the risk of radioactivity exposure and contamination during sampling in accordance with the ALARA principle (As Low As Reasonably Achievable) and implemented in a sampling glove box, routinely used to take samples from the basin and other watery environments (e.g., the primary and secondary cooling circuits). This also ensured that enough cell material could be collected for downstream analyses. Of note, filtration was chosen as a preferred method over centrifugation, as this resulted in a more effective and safer sample collection.

The system was composed of a stainless steel filter holder containing a $0,2 \mu \mathrm{m}$ pore sized polyethersulfone $\left(\right.$ Supor $^{\circledR}$ ) filter membrane (Pall Corporation, Port Washington, NY, United States), connected to the corresponding tap on one side and a flow meter on the other side in order to measure the filtered volume (Supplementary Figure 2). Ten liters of water were filtered per sample, after which the filter membrane was retrieved from the system and stored at $-20^{\circ} \mathrm{C}$ in a controlled area (for a period of 1-9 months) before DNA extraction to allow for radioactive decay.

\section{DNA Extraction and 16S rRNA Amplicon Sequencing}

DNA was extracted from the filter membranes following a previously described protocol (Vilchez-Vargas et al., 2013) and subsequently purified using Amicon ${ }^{\circledR} 30 \mathrm{kDa}$ filter cartridges (Merck, Darmstadt, Germany). DNA concentrations were measured using a Quantus ${ }^{\mathrm{TM}}$ fluorometer (Promega, Madison, WI, United States). For the 16S rRNA amplicon sequencing, the hypervariable V3-V4 region was chosen to specifically target bacteria (as opposed to fungi and/or Archaea) and the resulting amplicons were sequenced in two runs using V3 chemistry $(2 \times 300 \mathrm{bp})$ with the Illumina MiSeq platform (sequencing performed by Eurofins Genomics, Ebersberg, Germany). Positive (Cupriavidus metallidurans $\mathrm{CH} 34$ cultures filtered over the same filter membranes as previously mentioned) and negative controls (blank filter membranes and demineralized water) were also added as part of the experiment. They underwent the same DNA extraction procedure as the other samples. 


\section{Sequencing Data Analysis}

The data generated by the sequencing platform were demultiplexed and the datasets consisted of two separate fastq files (forward and reversed). The sequences were subsequently analyzed through the OCToPUS pipeline as described in Mysara et al. (2017): In short, reads went successively through preassembly denoising (Bankevich et al., 2012), contig assembly, quality filtering using mothur (Schloss et al., 2009), denoising using IPED (Mysara et al., 2016), chimera removal using CATCh (Mysara et al., 2015) and finally Operational Taxonomic Unit (OTU) clustering with 97\% cut-off using UPARSE (Edgar, 2013). All resulting OTUs were taken into account for further analyses without applying a minimum abundance threshold.

The OTUs were then taxonomically assigned using the mothur classify.seqs command with the RDP dataset (version $16)$ as reference. In order to properly compare the samples from both campaigns combined, they were rarefied to the same read count as the smallest sample (11,683 reads), using the mothur sub.sample command. Although a large number of reads is typically removed during this process, this does not significantly affect the subsequent alpha and beta diversity analyses. Rarefaction curves were obtained by using the mothur rarefaction.single command and subsequently plotting the data in the R software. Sample metadata are included in Supplementary Table 1.

Alpha (Inversed Simpson index) and beta [Theta-YC distance calculation followed by non-metric multi-dimensional scaling (NMDS)] diversity analyses were also performed in mothur using the summary.single, dist.shared, and nmds commands. In order to correlate the OTUs and metadata with the NMDS dimensions, a Spearman rank correlation analysis was performed using the mothur corr.axes command. OTUs and metadata that were significantly correlated $(r>|0.6|, p<0.05)$ with the represented NMDS axes are displayed on the NMDS plots. It must be noted that the structure of our data did not allow for the use of mixed models.

\section{Cell Counting}

During the second sampling campaign, a cell counting method was used to study the population dynamics of the basin water across the two reactor operation conditions (cycle vs. shutdown). The method consisted of heterotrophic plate count, where $100 \mu \mathrm{l}$ of basin water was directly spread onto R2A agar (Reasoner and Geldreich, 1985) plates in triplicates. The plates were incubated for 2 weeks at room temperature on a laboratory bench before colony counting. Flow cytometry was also considered as a cell counting method, but proved to be unreliable as the cell numbers during cycles dropped significantly, thereby making it impossible to distinguish between bacterial cells and instrument background. This meant that relative OTU abundances could not be corrected for absolute cell numbers.

\section{Physical Characterization of the Basin Environment}

The physical parameters of the BR2 waters are constantly monitored using on-line measuring instruments. Relevant data for the bacterial community (conductivity, temperature, radiation, and flow rate) were retrieved from an in-house software (BIDASSE) database and organized according to the sampling time points.

\section{Chemical Analysis}

Water samples from both cycles and shutdown periods from campaign 1 were collected to perform a chemical analysis of ecologically relevant compounds such as nitrate, phosphate, and carbon. The ions (nitrite, nitrate, phosphate, and acetate) were quantified through ion chromatography using a 930 Compact IC Flex (Metrohm, Herisau, Switzerland) with a Metrosep A Supp 16-250/4.0 column and a Metrosep A Supp 16 guard/4.0 column, using an eluent solution of $7.5 \mathrm{mM} \mathrm{Na}_{2} \mathrm{CO}_{3}$ and $0.75 \mathrm{mM} \mathrm{NaOH}$. TIC (total inorganic carbon) and TOC (total organic carbon) were measured using a FormacsHT-I TOC analyzer (Skalar Analytical, Breda, Netherlands). $\mathrm{O}_{2}, \mathrm{H}_{2}$, and ROS were not measured in our system.

\section{Temperature Experiment}

To assess the influence of temperature, another critical parameter, on the community, an experiment was designed to test its effect on cell number. Briefly, basin water was collected from the BR2 reactor during a shutdown period in an autoclaved glass container and incubated at $35^{\circ} \mathrm{C}$ (approximately the same temperature as observed during a cycle) for a period of $24 \mathrm{~h}$. As a control, the same volume of water was kept at room temperature $\left(21 \pm 2^{\circ} \mathrm{C}\right)$ next to the incubator. After $24 \mathrm{~h}$, a fresh sample was collected from the BR2 reactor (also at room temperature or $21 \pm 2^{\circ} \mathrm{C}$ ) in order to be compared with the control. Cell numbers for all three conditions were measured using a flow cytometry procedure: $100 \mathrm{ml}$ of basin water collected during shutdown was centrifuged at $17000 \times g$ for $30 \mathrm{~min}$, after which the supernatant was discarded and the (invisible) cell pellet re-suspended in $1 \mathrm{ml}$ of bottled Evian ${ }^{\circledR}$ water filtered over a $0.2 \mu \mathrm{m}$ Acrodisc syringe filter (Pall corporation). The solution was then stained with $\mathrm{SYBR}^{\circledR}$ green (Life Technologies, Carlsbad, CA, United States), incubated for $20 \mathrm{~min}$ at $37^{\circ} \mathrm{C}$ and run on an Accuri ${ }^{\mathrm{TM}} \mathrm{C} 6$ flow cytometer (BD Biosciences, San Jose, CA, United States) in four replicates of $200 \mu$ l. Cells were manually gated on green fluorescence (FL1) vs. size (FSC) plots based on a negative control $(0.2 \mu \mathrm{m}$ filtered Evian ${ }^{\circledR}$ water).

\section{RESULTS}

In order to study the dynamics of the bacterial community in the basin water, two sampling campaigns (separated by a 1-year interval) spanning multiple cycle and shutdown periods were performed using a $16 \mathrm{~S}$ rRNA amplicon sequencing approach. The physico-chemical parameters of the water were monitored across both campaigns, whereas the cell number was assessed only during the second campaign. Finally, an experiment was designed to assess the effect of temperature on the basin water cell number. 


\section{Physical Parameters}

During this study, four relevant physical parameters were monitored in order to potentially correlate those with the microbial community. The first parameter was the flow rate of the basin water cooling circuit (Figure 1A). During shutdown periods, the standard flow rate was $50 \mathrm{~m}^{3} \mathrm{~h}^{-1}$ while during operation it increased to $500 \mathrm{~m}^{3} \mathrm{~h}^{-1}$ to cool down the basin water. A second parameter was the global gamma radioactivity in the basin water (Figure 1B). As expected, the activity during operation significantly increased (up to a maximum of 9,500 cps)

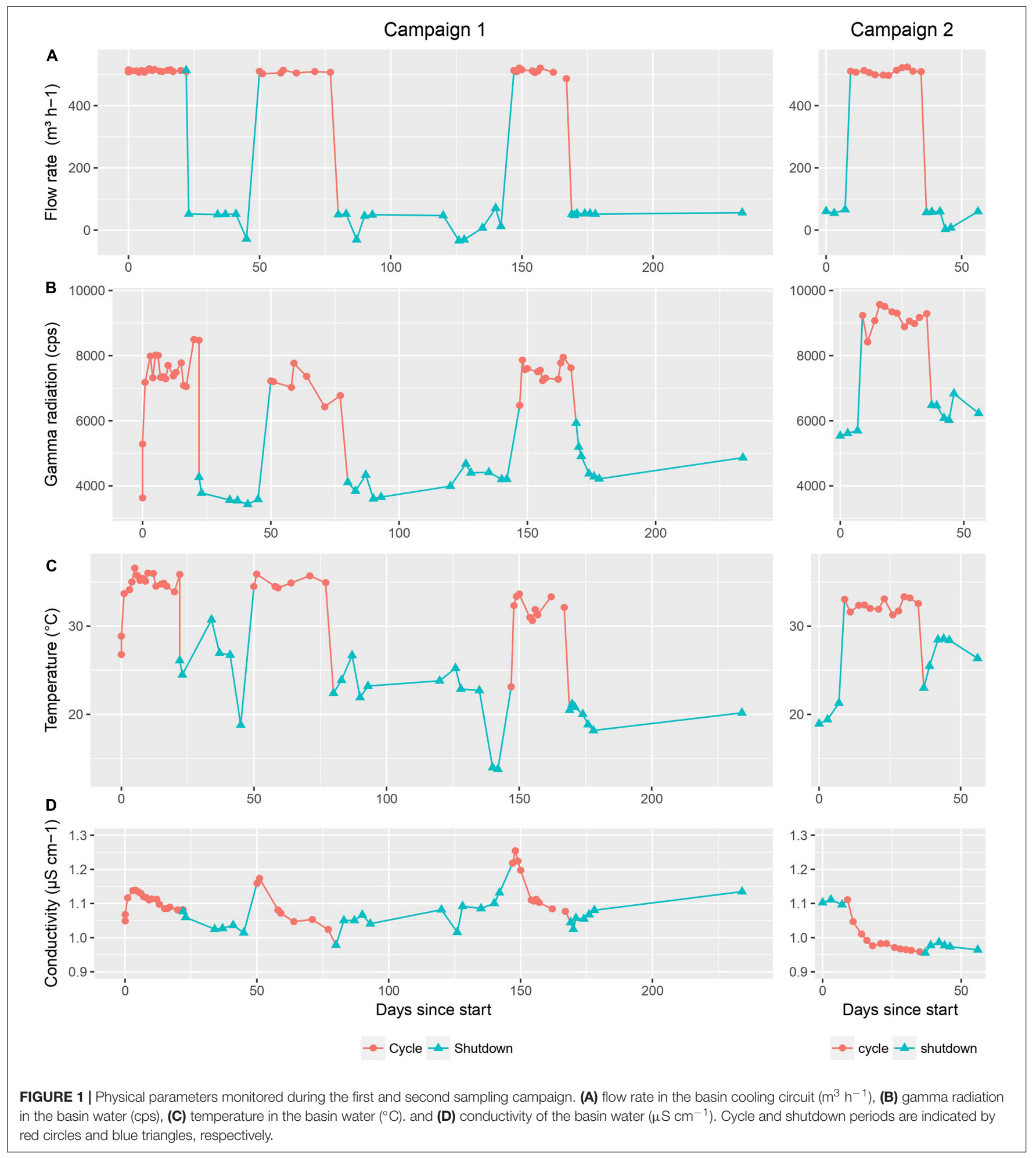


in comparison to shutdown periods (with a minimum of $3,500 \mathrm{cps}$ ) because of the nuclear fission reactions taking place in the reactor core. When the reactor is running, the radiation is deposited in the pool at a calculated dose rate of $755 \mathrm{~Gy} \mathrm{~h}^{-1}$, $98 \%$ of which is deposited in a zone within $5 \mathrm{~cm}$ around the core and $100 \mathrm{~cm}$ above and below the central plane. During shutdown periods, the dose rate lowers to $1 \mathrm{~Gy} \mathrm{~h}^{-1}$.

The third parameter was the temperature of the basin water (Figure 1C), which followed the same trend as observed for the other parameters: a significant increase during cycles (up to a maximum of $35^{\circ} \mathrm{C}$ ), followed by a decrease to previous levels during shutdowns (with a minimum of $15^{\circ} \mathrm{C}$ ). Lastly, the conductivity of the basin water also showed some variation across the timeline, although to a much smaller extent than the other parameters, with values ranging from 0.96 to $1.25 \mu \mathrm{S}$ $\mathrm{cm}^{-1}$ (Figure 1D). As a side note, the flow rate in the purification circuit where deionization happens through mixed bed ion exchange resins remained constant throughout cycles and shutdown periods with a value of $30 \mathrm{~m}^{3} \mathrm{~h}^{-1}$.

\section{Chemical Analysis}

The results of the chemical analysis performed on a selection of cycle and shutdown samples from campaign 1 can be found in Table 1. The concentrations of acetate and nitrate were extremely low, while the values for nitrite, phosphate, formate, TIC, and TOC were below the detection limit of the measuring instruments (10 $\mu \mathrm{g}^{-1}$ for ion chromatography and $500-700 \mu \mathrm{g} \mathrm{l}^{-1}$ for TIC-TOC analysis, respectively). Furthermore, no significant difference between cycle and shutdown values could be detected. Other elements such as $\mathrm{Zn}, \mathrm{Pb}, \mathrm{Co}, \mathrm{Ni}, \mathrm{Be}, \mathrm{Fe}, \mathrm{Mg}, \mathrm{Cu}, \mathrm{Al}, \mathrm{Ba}$, $\mathrm{Li}$, and $\mathrm{F}$ were also measured, but values were all below $20 \mu \mathrm{g}$ $1^{-1} \cdot \mathrm{pH}$ was maintained at an average value of $6 \pm 0.4$. Finally, the total gamma activity across campaign 1 and 2 amounted to $1.9 \times 10^{3} \mathrm{~Bq}^{-1}$ on average during cycles and $<80 \mathrm{~Bq}^{-1}$ during shutdowns. $95 \%$ of the activity during cycles originates from $\mathrm{Na}$ 24 , a radioisotope with a 15 -h half-life. The remainder comes from other radioisotopes such as Cr-51, Sb-124, Re-186, Np-239, W-188, and Co-60.

\section{Cell Counting}

In order to explore the effect of the changing physical conditions on the cell number of the bacterial population, heterotrophic

TABLE 1 | Chemical analysis performed on ecologically relevant compounds, during both cycle and shutdown periods.

\begin{tabular}{lcccc}
\hline Component & Cycle $\left(\boldsymbol{\mu} \mathbf{g ~ I}^{-\mathbf{1}}\right)$ & $\boldsymbol{n}$ & Shutdown $\left(\boldsymbol{\mu} \mathbf{~ I ~}^{-\mathbf{1}}\right)$ & $\boldsymbol{n}$ \\
\hline Acetate & $58 \pm 16$ & 5 & $67 \pm 33$ & 6 \\
Nitrate & $35 \pm 3$ & 5 & $44 \pm 22$ & 6 \\
Nitrite & $<10$ & 5 & $<10$ & 6 \\
Phosphate & $<10$ & 5 & $<10$ & 6 \\
Formate & $<10$ & 5 & $<10$ & 6 \\
TIC & $<500$ & 3 & $<500$ & 3 \\
TOC & $<700$ & 3 & $<700$ & 3 \\
\hline
\end{tabular}

Values represent mean \pm standard deviation. plate count was used during the second sampling campaign (Figure 2). The data for this method follow a clear pattern: a steep decline in cell number of $1.2 \log _{10} \mathrm{CFU} \mathrm{ml} \mathrm{m}^{-1}$ at the start of the cycle, followed by a stabilization and slow increase of the population by $0.5 \log _{10} \mathrm{CFU} \mathrm{m} \mathrm{m}^{-1}$ during the cycle and finally a recovery to previous cell density levels (up to $3.3 \log _{10} \mathrm{CFU} \mathrm{ml}{ }^{-1}$ ) after the cycle.

The observed pattern of cell number can be correlated with the change in physico-chemical parameters described previously. During a cycle, the flow rate of the cooling circuit for the basin water goes up from 50 to $500 \mathrm{~m}^{3} \mathrm{~h}^{-1}$. After passing through this circuit, the water is transferred back into the basin via a conduit following the reactor wall. At this point, the water is exposed to high levels of radiation originating from the nuclear fission taking place in the reactor core (gamma heating of $0.83 \mathrm{~W}$ $\mathrm{g}^{-1}=830 \mathrm{~Gy} \mathrm{~s}^{-1}$ for a representative cycle of operation). Due to the high flow rate of the cooling circuit during a cycle, the water is frequently exposed to this radiation, with a turnover time of ca. $104 \mathrm{~min}$.

\section{Bacterial Community Structure}

The summary of the data analysis for the basin water samples given in Table 2 shows that the average number of OTUs for both campaigns did not significantly differ across cycles and shutdowns. Rarefaction curves for all samples are shown in Supplementary Figure 3.

Figure 3 shows the 10 most abundant OTUs in the basin water, representing 90 and $76 \%$ of the total reads for campaign 1 and 2 , respectively. A clear shift in the community profile across the different cycle and shutdown periods is apparent. During the first sampling campaign, the most abundant OTU in the first two cycles was assigned to an unclassified Gammaproteobacterium (OTU5), accounting for $28 \%$ of total amplicon reads. For the third cycle, an OTU assigned to Pelomonas (OTU2) became predominant at $43 \%$, although it could already be observed in lower proportions, at 5\%, during the previous cycles. During the shutdown periods in between the cycles, the community profile shifted drastically and became dominated by an OTU assigned to Methylobacterium (OTU3) at 41\%, while the previously mentioned OTUs (OTU5 and OTU2) were detected in much lower proportions (3 and 7\%, respectively). This change can probably be assigned to the shift in physico-chemical parameters that occurs with the transition from a cycle to a shutdown period.

Although the second sampling campaign took place 1 year after the first one, the same pattern could be observed, indicating that the system is rather stable. Nevertheless, a difference can be noted for the unclassified Gammaproteobacterium and Pelomonas observed in approximately equal proportions (13 and $10 \%$, respectively), whereas the community was either dominated by one or the other during the first sampling campaign.

\section{Temperature Effect on Cell Number}

As can be observed in Figure 4, the cell number significantly increased when exposed to a higher temperature, indicating that temperature has an opposite effect to radiation. Since the overall cell number decreases during a cycle, this shows that the effect of radiation exposure largely outperforms the effect of temperature. 


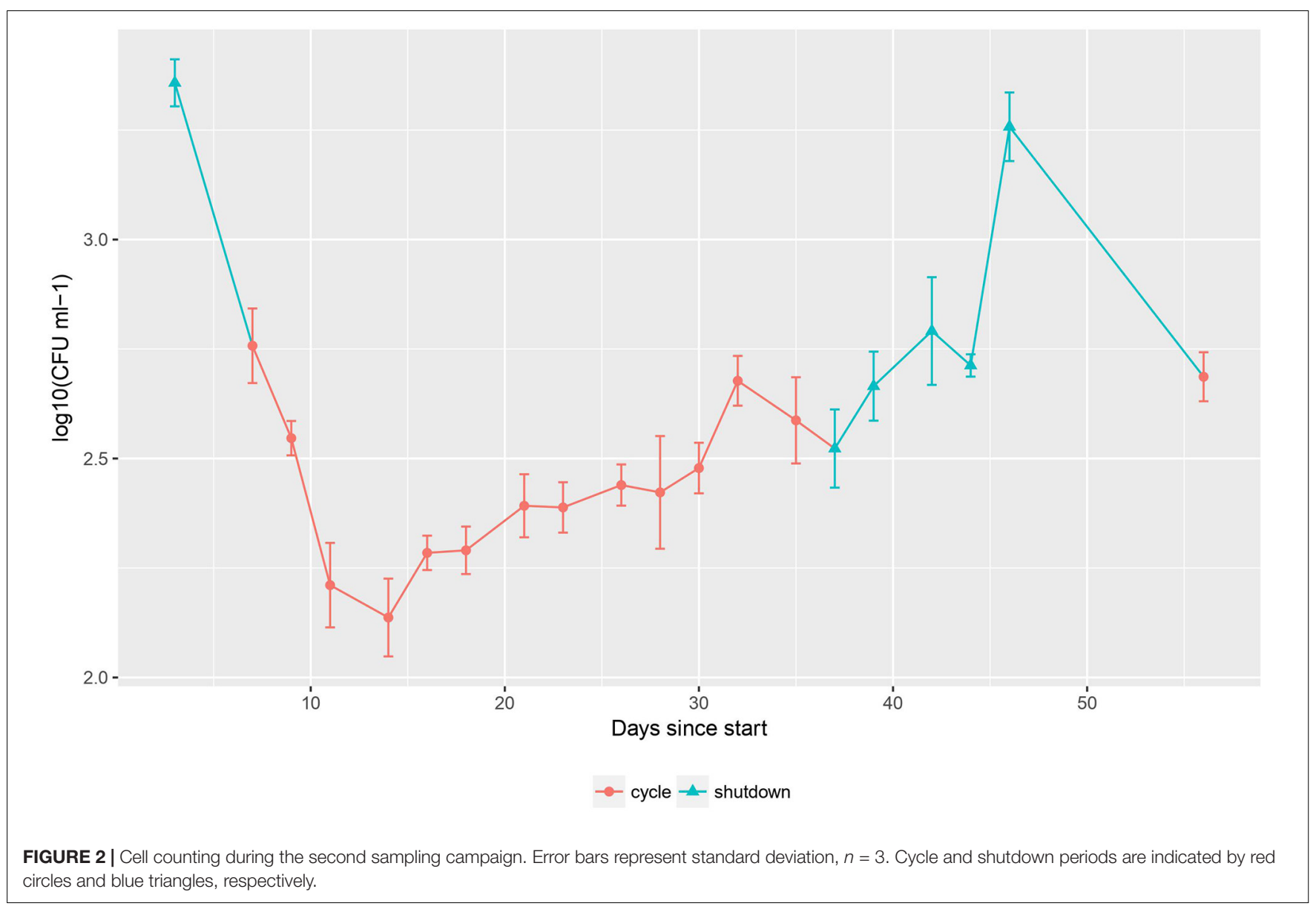

\section{Alpha and Beta Diversity Analyses}

The alpha diversity showed a peak in diversity at the start of each cycle, followed by a subsequent downward trend (Supplementary Figure 4). This was true for both sampling campaigns, which further confirmed the robustness of the system under study.

For the beta diversity analysis, an NMDS was performed on all the samples using the mothur program (Schloss et al., 2009). As can be observed in Figure 5, all shutdown samples

TABLE 2 | Summary of the data analysis performed for the first and second sampling campaigns.

\begin{tabular}{lcc}
\hline & Campaign 1 & Campaign 2 \\
\hline Sampling dates & $06 / 09 / 2016-$ & $16 / 04 / 2018-$ \\
& $28 / 04 / 2017$ & $11 / 06 / 2018$ \\
Number of samples & 61 & 21 \\
Total number of reads & $6,127,782$ & $4,20,191$ \\
Average number of reads per sample & $1,00,455$ & 20,009 \\
Minimum & 55,044 & 11,683 \\
Maximum & $1,50,145$ & 28,867 \\
Standard deviation & 18,990 & 3,611 \\
Total number of OTUs & 4,017 & 864 \\
Average number of OTUs per sample & 225 & 172
\end{tabular}

from the first campaign clustered together, indicating that the community returns to an equilibrium after each cycle. Cycles 1 and 2 also clustered together in the top right quadrant, whereas cycle 3 was clustered separately in the bottom right quadrant, indicating a shift in the community at this point in time. This was also confirmed in Figure 3. Furthermore, the data points for the second campaign can be observed to blend in with the data points from campaign 1. Taking into consideration that the sampling campaigns were approximately 1 year apart, this further corroborates the fact that the system is very stable. Indeed, the shutdown data points for both campaigns were clustered together in the two left quadrants, whereas the cycle data points were clustered in the two right quadrants. Furthermore, the cycle from campaign 2 intercalates in between the cycles from the previous campaign, which supports a model where the bacterial community oscillates between different states.

In order to evaluate which parameters were responsible for the community shifts, a Spearman rank correlation analysis was performed between the data points on the NMDS axes and the observed OTUs and physico-chemical parameters. The OTUs with the largest impact on the separation between cycle and shutdown samples are represented as blue arrows in Figure 5. Regarding the physico-chemical parameters, it was observed that gamma radiation, flow rate, and temperature showed a significant correlation with the horizontal axis, whereas conductivity was 


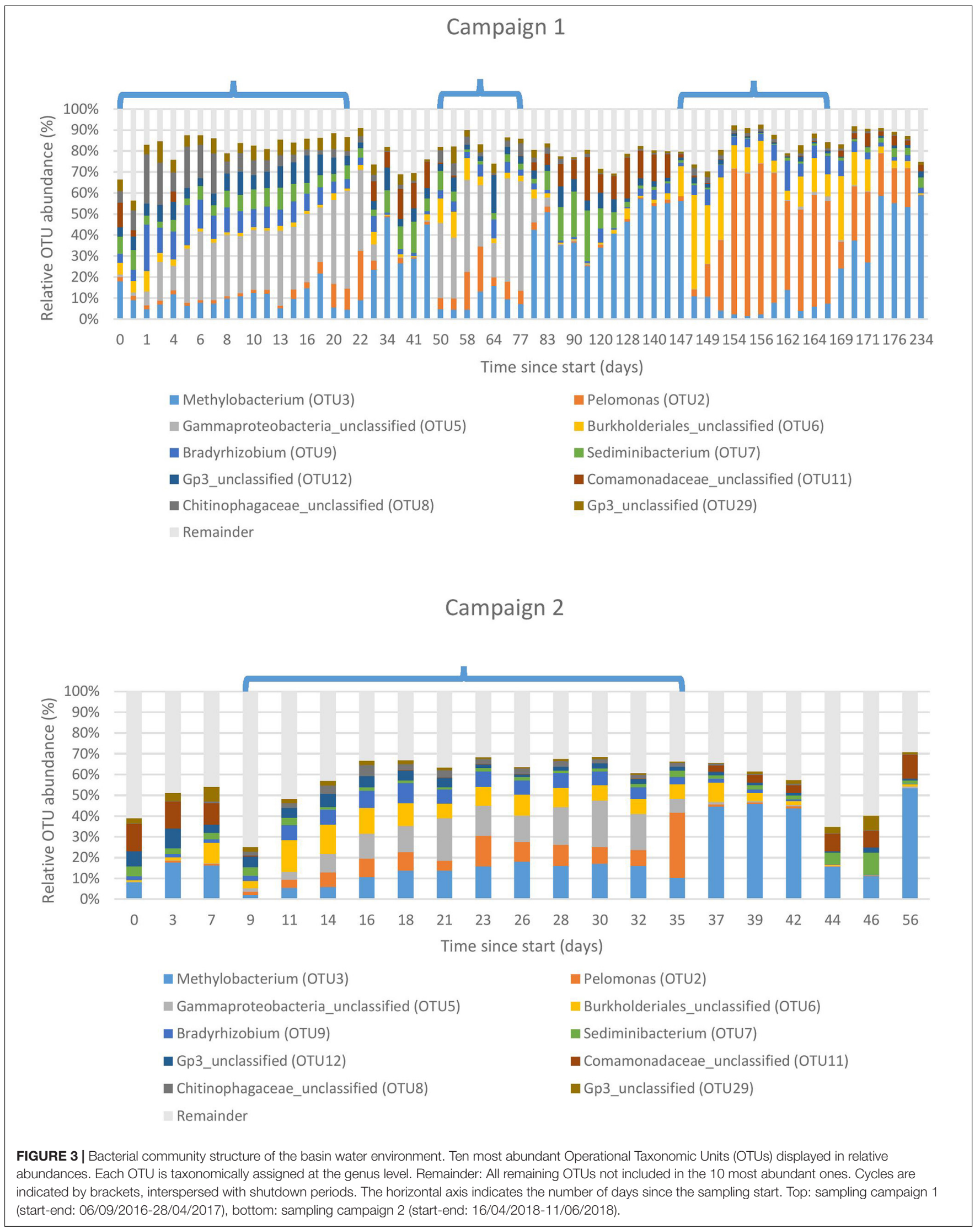


Effect of temperature on basin water cell number

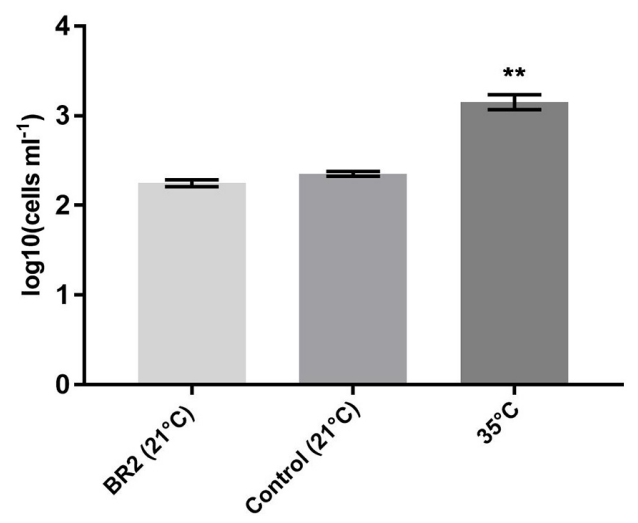

FIGURE 4 | Effect of 24-h temperature increase on basin water cell number. Error bars represent standard error of the mean. Groups were compared using a non-parametric Kruskal-Wallis test. $P$-value $=0.0024, n=4,{ }^{\star \star} p<0.01$.

marginally significant. Acetate and nitrate concentrations did not show a significant correlation. This suggests that radiation exposure, flow rate and temperature could be important factors in the community shift observed across cycles and shutdown periods. Correlation coefficients and $p$-values for the described OTUs and physico-chemical parameters can be found in Table 3.

\section{DISCUSSION}

The aim of the present study was to explore the bacterial community dynamics in the basin surrounding the core vessel of a nuclear research reactor. A first relevant observation was that the communities in the basin water underwent an apparent shift across cycle and shutdown periods. This was clearly observed in the community profiles and was further confirmed by the NMDS analysis, in which shutdown and cycle samples clustered separately. This shift correlated with a change in physico-chemical parameters such as temperature, flow rate and gamma radiation, whereas conductivity, nitrate, and acetate showed no significant correlation, indicating that the latter might have a lesser or no influence on the community composition. Other parameters such as $\mathrm{pH}$ and other ions were not included as these were either maintained at stable levels throughout the entire study or below the detection limit of the measuring instruments. The relevant parameters (individually or in combination) probably had an impact on the community shift from a population dominated by a member of the Gammaproteobacteria and Pelomonas during cycles to one dominated by Methylobacterium during shutdown periods. This pattern was observed for both sampling campaigns, indicating that this periodicity is stable. However, additional research is needed to elucidate the role of each individual parameter on the community, since all three of them follow the same pattern (high values during cycles and low values during shutdowns).

As such, temperature and radiation are believed to have the largest stress impact on the community. The observed shift during a transition from a shutdown period to a cycle could also be facilitated by the effect of stress on the community. Indeed, it has been previously described that the level of invasion of new species in communities with identical evenness depends strictly on the presence of stress (De Roy et al., 2013), here in the form of radiation exposure. In the absence of stress, communities tend to be more resistant to invasion.

The most abundant OTU during the third cycle of the first sampling campaign was Pelomonas, as opposed to the previous cycles that were mostly dominated by an unclassified Gammaproteobacterium. However, the cycle monitored during the second sampling campaign showed an approximately equal prevalence of both the unclassified Gammaproteobacterium and Pelomonas. This is reflected in the NMDS analysis, where the data points of the campaign 2 cycle intercalate in the middle of the other cycle data points with little overlap. This suggests that the community during cycles can fluctuate between different states, either equally dominated by the two main OTUs or only dominated by one of them. Taking into consideration that the physico-chemical parameters across different cycles remain constant, this phenomenon can probably be attributed to stochastic effects, which might be investigated in future research. Indeed, it is known that the mechanisms governing community assembly can be both deterministic and stochastic in nature (Sloan et al., 2006; Nemergut et al., 2013; Evans et al., 2017; Zhou and Ning, 2017). As such, next to deterministic factors like species traits, interspecies interactions and environmental conditions, stochastic processes in the form of random birth and death events, colonization and extinction are also known to play a role in this regard. Furthermore, it has been shown that a single source community can, under identical environmental conditions, generate drastically different communities with little overlap in composition or even global function (Zhou et al., 2013).

The chemical analysis of the water samples showed that all of the measured components (nitrite, phosphate, formate, TIC, and TOC as well as other chemical elements) except for acetate and nitrate were below the detection limit of the measuring instruments. This is due to the fact that the water is constantly being deionized through ion exchangers, resulting in a very low conductivity of approximately $1 \mu \mathrm{S} \mathrm{cm}^{-1}$ and an extremely oligotrophic environment. However, even in ultrapure waters, it is impossible to eliminate all impurities. In environments such as the one monitored in our study, these impurities mainly originate from metallic pipings in the cooling and purification circuits, dust that falls into the basin and the air in contact with the pool surface. Typical dominant impurities include $\mathrm{Al}_{3}{ }^{+}, \mathrm{Fe}_{2}{ }^{+}$, $\mathrm{NO}_{3}{ }^{-}, \mathrm{SO}_{42}{ }^{-}$and $\mathrm{Cl}^{-}$(International Atomic Energy Agency, 2011). These can in turn be utilized by bacterial communities. Furthermore, the basin of the BR2 research reactor is regularly emptied during shutdown periods for maintenance purposes, allowing technicians to access areas that would normally be submerged. This could periodically introduce organic material in the system, which could be used as an energy source by heterotrophic bacteria.

Another source of organic material could be the ion exchange resins used in the purification circuit. These are mixed bed resins composed of polystyrene beads that absorb cations and anions on 


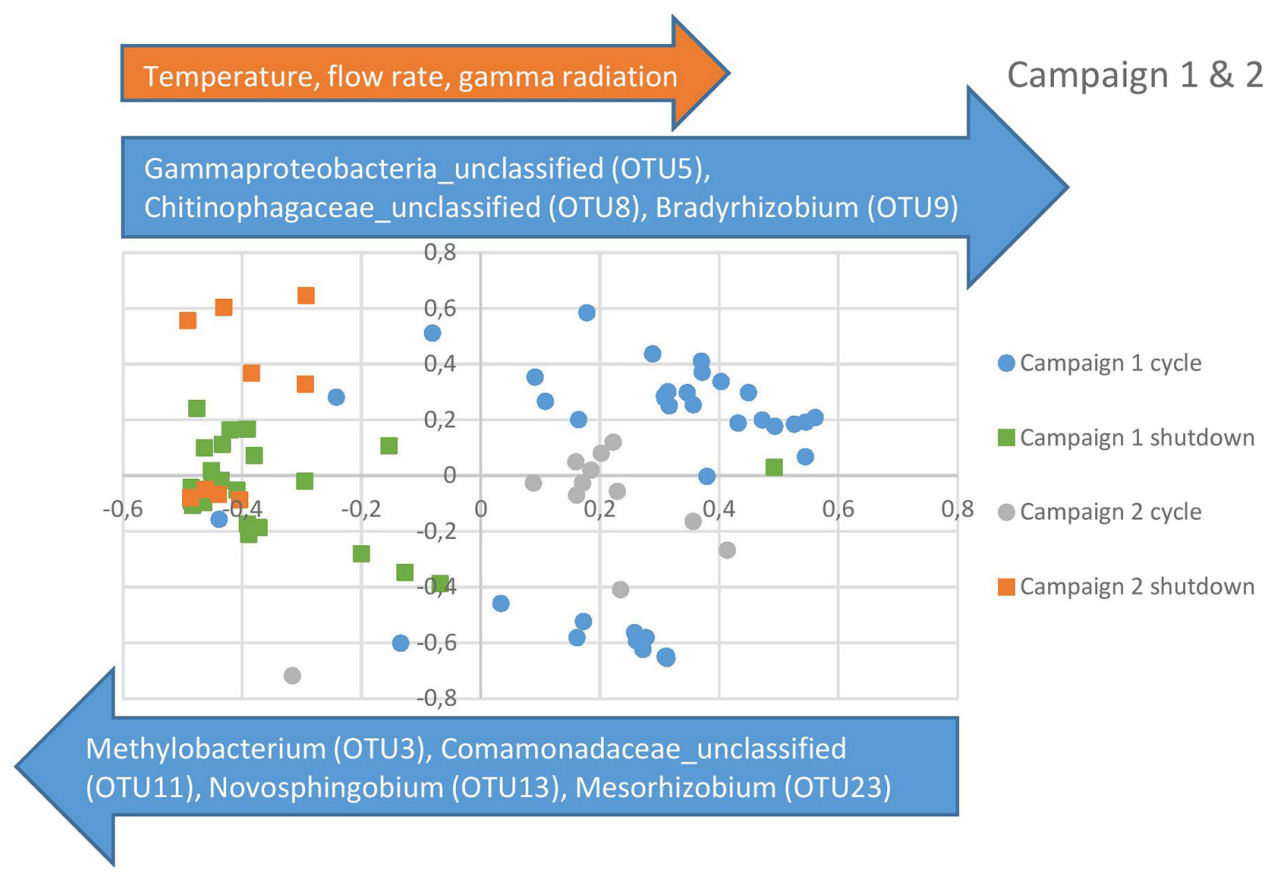

FIGURE 5 | Non-metric Multidimensional Scaling (NMDS) for campaign 1 and 2 combined. The blue and orange arrows, respectively represent the OTUs and physico-chemical parameters showing a significant correlation $(r>0.6, p<0.05)$ with the represented NMDS axes.

their surface. Due to radiation, these resins slowly degrade over time (approximately 5 years), hereby releasing small quantities of organic material into the water, which can then be used by bacteria. In addition, they could also be a substrate for bacterial growth, as has been demonstrated by one particular research group (Kéki et al., 2019).

The basin is an oxic environment, since it is an open system with high water circulation $\left(50-500 \mathrm{~m}^{3} \mathrm{~h}^{-1}\right)$ which allows for a homogeneous oxygen distribution. This is also evidenced by the fact that most identified genera based on the $16 \mathrm{~S}$ rRNA amplicon sequencing data are strictly or facultatively aerobic. In addition, the presence of ionizing radiation causes water molecules to dissociate into several chemical species such as $\mathrm{H}_{2}, \mathrm{H}_{2} \mathrm{O}_{2}$, and some radicals $\left(\mathrm{OH} \bullet, \mathrm{H} \bullet\right.$, and $\left.\mathrm{HO}_{2} \bullet\right)$, a phenomenon known as water radiolysis (Dzaugis et al., 2015). These characteristics combined with the low nutrient availability

TABLE 3 | Summary of Spearman rank correlation analysis performed on Operational Taxonomic Units (OTUs) and physico-chemical parameters represented on the Non-metric Multidimensional Scaling (NMDS) plot.

\begin{tabular}{lcccccc}
\hline OTU & Average abundance & $\boldsymbol{r}$ & $\boldsymbol{p}$-value & Parameter & $\boldsymbol{r}$ & $\boldsymbol{p}$-value \\
\hline OTU5 & $11.39 \%$ & 0.77 & 0 & Temperature & 0.71 & 0 \\
OTU8 & $3.40 \%$ & 0.70 & 0 & Flow rate & 0.63 & 0 \\
OTU9 & $5.53 \%$ & 0.62 & 0 & Gamma radiation & 0.62 & 0 \\
OTU3 & $21.20 \%$ & -0.80 & 0 & Conductivity & 0.22 & 0.05 \\
OTU11 & $3.76 \%$ & -0.84 & 0 & Acetate & 0.50 & 0.16 \\
OTU13 & $2.06 \%$ & -0.69 & 0 & Nitrate & 0.32 & 0.37 \\
OTU23 & $1.04 \%$ & -0.81 & 0 & & &
\end{tabular}

result in some unique conditions for which different bacteria have adopted different strategies. For example, it has been previously demonstrated that bacteria in SNFPs can oxidize $\mathrm{H}_{2}$ from water radiolysis as an energy source, using $\mathrm{O}_{2}$ as electron acceptor and $\mathrm{CO}_{2}$ as carbon source (Galès et al., 2004). As such, the Pelomonas and Bradyrhizobium genera as well as the Comamonadaceae family detected in our system have been associated with chemoautotrophic growth on $\mathrm{H} 2$ (Willems et al., 1991; Gomila et al., 2007; Franck et al., 2008). On the other hand, Methylobacterium is a facultative methylotroph known to be able to grow on C2 compounds such as acetate as a sole carbon source (Smejkalova et al., 2010; Green and Ardley, 2018). Bradyrhizobium and Mesorhizobium on the other hand are capable of $\mathrm{N}_{2}$ fixation (Black et al., 2012). It must also be noted that bacteria in oligotrophic environments can rely on mixotrophy as a survival strategy, since this provides them with a greater nutritional flexibility (Mittelman and Jones, 2018). Furthermore, the above-described taxa were also detected in different SNFPs and other cooling basins using 16S rRNA amplicon sequencing (Bagwell et al., 2018; MeGraw et al., 2018; Petit, 2018; Petit et al., 2020). This can probably be attributed to the fact that the physico-chemical parameters in SNFPs and the basin under study are quite similar. Indeed, the water in SNFPs is also very oligotrophic due to constant deionization via anion and cation exchangers and is exposed to residual levels of radiation originating from the spent fuel elements stored underwater.

In addition, we propose that the established community may be able to recycle dead cell material in order to survive long periods of nutrient deprivation. Indeed, it has been previously 
demonstrated that bacteria can survive for extended periods of time under conditions of starvation, and that this phenomenon is inversely proportional to cell density (Phaiboun et al., 2015). Moreover, bacteria in their natural environments are believed to exist in conditions resembling those of long-term stationary phase cultures, where stress-response genes and alternative metabolic pathways are essential for survival (Finkel, 2006). It has also been shown that cell death and recycling were essential processes for cell survival during starvation, where survivors were able to utilize nutrients from dead cells parsimoniously as a strategy for long-term persistence (Takano et al., 2017). In our system, the cell number rapidly decreases with a magnitude of more than one log during the transition from a shutdown period to a cycle (Figure 2). This cell number decrease could be assigned to the water being exposed to high levels of radiation originating from the reactor core. We therefore propose that cells that have been killed off by radiation are rapidly being recycled by the surviving population for nutrient retrieval. This phenomenon could also partially explain the peak in diversity observed at the start of each cycle (Supplementary Figure 4). Indeed, the temporary availability in nutrients could allow other species to emerge, resulting in a brief increase in both richness and evenness before the community reverts toward an equilibrium state characterized by a lower diversity. The stressing effect of radiation on the community could also account for the downward trend in diversity observed for both sampling campaigns. This could potentially be investigated in further research.

In this study, we mainly focused on the characterization of the bacterial community, but it could also be of interest to investigate the presence of eukaryotes such as microalgae and fungi through $18 \mathrm{~S}$ and ITS amplicon sequencing. Our study could also be complemented with additional research investigating the radiation resistance of individual strains isolated from this environment or of the entire community as a whole. In addition, it would be of interest to perform a deeper analysis on the community using NGS techniques such as shotgun metagenomics and metaproteomics. This would allow for the development of a deeper insight into the specific genes and proteins responsible for the behavior of the community in this unique environment.

\section{REFERENCES}

Bagwell, C. E., Noble, P. A., Milliken, C. E., Li, D., and Kaplan, D. I. (2018). Amplicon sequencing reveals microbiological signatures in spent nuclear fuel storage basins. Front. Microbiol. 9:377. doi: 10.3389/fmicb.2018.00377

Bankevich, A., Nurk, S., Antipov, D., Gurevich, A. A., Dvorkin, M., Kulikov, A. S. et al. (2012). SPAdes: a new genome assembly algorithm and its applications to single-cell sequencing. J. Comput. Biol. 19, 455-477. doi: 10.1089/cmb.2012. 0021

Black, M., Moolhuijzen, P., Chapman, B., Barrero, R., Howieson, J., Hungria, M., et al. (2012). The genetics of symbiotic nitrogen fixation: comparative genomics of 14 rhizobia strains by resolution of protein clusters. Genes (Basel) 3, 138-166. doi: 10.3390/genes3010138

Chicote, E., Garcia, A. M., Moreno, D. A., Sarro, M. I., Lorenzo, P. I., and Montero, F. (2005). Isolation and identification of bacteria from spent nuclear fuel pools. J. Indust. Microbiol. Biotechnol. 32, 155-162. doi: 10.1007/s10295-005-0216-3

\section{DATA AVAILABILITY STATEMENT}

The datasets presented in this study can be found online in the BioProject repository (https://www.ncbi.nlm.nih.gov/ bioproject/) under accession number PRJNA725077.

\section{AUTHOR CONTRIBUTIONS}

VVE performed the sampling, laboratory work and data analysis, and wrote the manuscript. RP provided advice on the research design and flow cytometry. MM contributed to the 16SrRNA sequencing data processing. PM provided advice on the research design and data interpretation. All authors critically reviewed the manuscript.

\section{FUNDING}

This research was funded by the SCK CEN Academy.

\section{ACKNOWLEDGMENTS}

We thank Hans Ooms, Dirk Meynen, Emre Sikik, Bart Thijs, and Steven Van Dyck for providing valuable information about the BR2 reactor and the radiation control department for verifying sample activity. We also thank Robby Nijs, Patrick Claes, Job Cools, Eddy Kox, and May Van Hees for ensuring all lab work involving radioactivity was performed according to ALARA regulations, Hugo Moors for providing advice on filtration methodologies, and Rob Van Houdt for providing advice on the manuscript structure.

\section{SUPPLEMENTARY MATERIAL}

The Supplementary Material for this article can be found online at: https://www.frontiersin.org/articles/10.3389/fmicb. 2021.744115/full\#supplementary-material

De Roy, K., Marzorati, M., Negroni, A., Thas, O., Balloi, A., Fava, F., et al. (2013). Environmental conditions and community evenness determine the outcome of biological invasion. Nat. Commun. 4:1383. doi: 10.1038/ncomms2392

Dzaugis, M. E., Spivack, A. J., and D'hondt, S. (2015). A quantitative model of water radiolysis and chemical production rates near radionuclide-containing solids. Radiat. Phys. Chem. Oxf. Engl. 1993 115, 127-134. doi: 10.1016/j.radphyschem. 2015.06.011

Edgar, R. C. (2013). UPARSE: highly accurate OTU sequences from microbial amplicon reads. Nat. Methods 10, 996-998. doi: 10.1038/nmeth.2604

Evans, S., Martiny, J. B. H., and Allison, S. D. (2017). Effects of dispersal and selection on stochastic assembly in microbial communities. ISME J. 11, 176185. doi: 10.1038/ismej.2016.96

Finkel, S. E. (2006). Long-term survival during stationary phase: evolution and the GASP phenotype. Nat. Rev. Microbiol. 4, 113-120. doi: 10.1038/nrmicro1340

Foster, L., Boothman, C., Ruiz-Lopez, S., Boshoff, G., Jenkinson, P., Sigee, D., et al. (2020). Microbial bloom formation in a high $\mathrm{pH}$ spent nuclear 
fuel pond. Sci. Total Environ. 720:137515. doi: 10.1016/j.scitotenv.2020. 137515

Franck, W. L., Chang, W. S., Qiu, J., Sugawara, M., Sadowsky, M. J., Smith, S. A., et al. (2008). Whole-genome transcriptional profiling of Bradyrhizobium japonicum during chemoautotrophic growth. J. Bacteriol. 190, 6697-6705. doi: 10.1128/JB.00543-08

Galès, G., Libert, M., Sellier, R., Cournac, L., Chapon, V., and Heulin, T. (2004). Molecular hydrogen from water radiolysis as an energy source for bacterial growth in a basin containing irradiating waste. FEMS Microbiol. Lett. 240, 155-162. doi: 10.1016/j.femsle.2004.09.025

Giacobone, A. F. F., Rodriguez, S. A., Burkart, A. L., and Pizarro, R. A. (2011). Microbiological induced corrosion of AA 6061 nuclear alloy in highly diluted media by Bacillus cereus RE 10. Int. Biodeter. Biodegr. 65, 1161-1168. doi: 10.1016/j.ibiod.2011.08.012

Gomila, M., Bowien, B., Falsen, E., Moore, E. R., and Lalucat, J. (2007). Description of Pelomonas aquatica sp. nov. and Pelomonas puraquae sp. nov., isolated from industrial and haemodialysis water. Int. J. Syst. Evol. Microbiol. 57, 2629-2635. doi: 10.1099/ijs.0.65149-0

Green, P. N., and Ardley, J. K. (2018). Review of the genus Methylobacterium and closely related organisms: a proposal that some Methylobacterium species be reclassified into a new genus, Methylorubrum gen. nov. Int. J. Syst. Evol. Microbiol. 68, 2727-2748. doi: 10.1099/ijsem.0.002856

International Atomic Energy Agency (2011). Good Practices for Water Quality Management in Research Reactors and Spent Fuel Storage Facilities. IAEA Nuclear Energy Series No. NP-T-5.2. Vienna: International Atomic Energy Agency.

Jolley, D. M. (2002). Radionuclide uptake and transport on microbes in potential repository drifts at Yucca Mountain, Nevada. Sci. Basis Nuclear Waste Manage. Xxv 713, 767-774.

Kéki, Z., Makk, J., Barkács, K., Vajna, B., Palatinszky, M., Márialigeti, K., et al. (2019). Critical point analysis and biocide treatment in a microbiologically contaminated water purification system of a power plant. SN Appl. Sci. 1:820.

Masurat, P., Fru, E. C., and Pedersen, K. (2005). Identification of Meiothermus as the dominant genus in a storage system for spent nuclear fuel. J. Appl. Microbiol. 98, 727-740. doi: 10.1111/j.1365-2672.2004.02519.x

MeGraw, V. E., Brown, A. R., Boothman, C., Goodacre, R., Morris, K., Sigee, D., et al. (2018). A novel adaptation mechanism underpinning algal colonization of a nuclear fuel storage pond. $m$ Bio 9:e02395-17. doi: 10.1128/mBio.02395-17

Mittelman, M. W., and Jones, A. D. G. (2018). A pure life: the microbial ecology of high purity industrial waters. Microb. Ecol. 76, 9-18. doi: 10.1007/s00248-0160736-6

Mysara, M., Leys, N., Raes, J., and Monsieurs, P. (2016). IPED: a highly efficient denoising tool for Illumina MiSeq Paired-end 16S rRNA gene amplicon sequencing data. BMC Bioinform. 17:192. doi: 10.1186/s12859-016-1061-2

Mysara, M., Njima, M., Leys, N., Raes, J., and Monsieurs, P. (2017). From reads to operational taxonomic units: an ensemble processing pipeline for MiSeq amplicon sequencing data. Gigascience 6:10. doi: 10.1093/gigascience/giw017

Mysara, M., Saeys, Y., Leys, N., Raes, J., and Monsieurs, P. (2015). CATCh, an ensemble classifier for chimera detection in $16 \mathrm{~S}$ rRNA sequencing studies. Appl. Environ. Microbiol. 81, 1573-1584. doi: 10.1128/AEM.02896-14

Nemergut, D. R., Schmidt, S. K., Fukami, T., O’neill, S. P., Bilinski, T. M., Stanish, L. F., et al. (2013). Patterns and processes of microbial community assembly. Microbiol. Mol. Biol. Rev. 77, 342-356. doi: 10.1128/MMBR.00051-12

Petit, P. C. M. (2018). Inventory of Microbiological Species Living in Spent Nuclear Fuel Pools: Towards The Identification of Radioresistant Species. Ph.D. thesis. Grenoble: Université Grenoble Alpes.

Petit, P. C. M., Pible, O., Van Eesbeeck, V., Alban, C., Steinmetz, G., Mysara, M., et al. (2020). Direct Meta-analyses reveal unexpected microbial life in the highly radioactive water of an operating nuclear reactor core. Microorganisms 8:1857. doi: 10.3390/microorganisms8121857

Phaiboun, A., Zhang, Y., Park, B., and Kim, M. (2015). Survival kinetics of starving bacteria is biphasic and density-dependent. PLoS Comput. Biol. 11:e1004198. doi: 10.1371/journal.pcbi.1004198

Reasoner, D. J., and Geldreich, E. E. (1985). A new medium for the enumeration and subculture of bacteria from potable water. Appl. Environ. Microbiol. $49,1-7$.

Rivasseau, C., Farhi, E., Compagnon, E., Saint Cyr, D. D., Van Lis, R., Falconet, D., et al. (2016). Coccomyxa actinabiotis sp nov (Trebouxiophyceae, Chlorophyta), a new green microalga living in the spent fuel cooling pool of a nuclear reactor. J. Phycol. 52, 689-703. doi: 10.1111/jpy.12442
Ruiz-Lopez, S., Foster, L., Boothman, C., Cole, N., Morris, K., and Lloyd, J. R. (2020). Identification of a stable hydrogen-driven microbiome in a highly radioactive storage facility on the sellafield site. Front. Microbiol. 11:587556. doi: $10.3389 /$ fmicb.2020.587556

Santo Domingo, J. W., Berry, C. J., Summer, M., and Fliermans, C. B. (1998). Microbiology of spent nuclear fuel storage basins. Curr. Microbiol. 37, 387-394.

Schloss, P. D., Westcott, S. L., Ryabin, T., Hall, J. R., Hartmann, M., Hollister, E. B., et al. (2009). Introducing mothur: open-source, platform-independent, community-supported software for describing and comparing microbial communities. Appl. Environ. Microb. 75, 7537-7541. doi: 10.1128/Aem. 01541-09

Sloan, W. T., Lunn, M., Woodcock, S., Head, I. M., Nee, S., and Curtis, T. P. (2006). Quantifying the roles of immigration and chance in shaping prokaryote community structure. Environ. Microbiol. 8, 732-740. doi: 10.1111/j.1462-2920. 2005.00956.x

Smart, N. R., Rance, A. P., Reddy, B., Hallbeck, L., Pedersen, K., and Johansson, A. J. (2014). In situ evaluation of model copper-cast iron canisters for spent nuclear fuel: a case of microbiologically influenced corrosion (MIC). Corrosion Eng. Sci. Technol. 49, 548-553. doi: 10.1179/1743278214y.0000000213

Smejkalova, H., Erb, T. J., and Fuchs, G. (2010). Methanol assimilation in Methylobacterium extorquens AM1: demonstration of all enzymes and their regulation. PLoS One 5:e13001. doi: 10.1371/journal.pone.001 3001

Takano, S., Pawlowska, B. J., Gudelj, I., Yomo, T., and Tsuru, S. (2017). Densitydependent recycling promotes the long-term survival of bacterial populations during periods of starvation. mBio 8:e02336-16. doi: 10.1128/mBio.02336-16

Tisakova, L., Pipiska, M., Godany, A., Hornik, M., Vidova, B., and Augustin, J. (2013). Bioaccumulation of Cs- 137 and Co- 60 by bacteria isolated from spent nuclear fuel pools. J. Radioanal. Nuclear Chem. 295, 737-748. doi: 10.1007/ s10967-012-1932-6

Vilchez-Vargas, R., Geffers, R., Suarez-Diez, M., Conte, I., Waliczek, A., Kaser, V. S., et al. (2013). Analysis of the microbial gene landscape and transcriptome for aromatic pollutants and alkane degradation using a novel internally calibrated microarray system. Environ. Microbiol. 15, 1016-1039. doi: 10.1111/j.14622920.2012.02752.x

Whitman, W. B., Coleman, D. C., and Wiebe, W. J. (1998). Prokaryotes: the unseen majority. Proc. Natl. Acad. Sci. U.S.A. 95, 6578-6583. doi: 10.1073/pnas.95.12. 6578

Willems, A., De Ley, J., Gillis, M., and Kerster, K. (1991). Comamonadaceae, a new family encompassing the Acidovorans rRNA complex, including Variovorax paradoxus gen. nov., comb. nov., for Alcaligenes paradoxus (Davis 1969). Int. J. Syst. Bacteriol. 41, 445-450.

Zhang, H. J., Dirk, W. J., and Geesey, G. G. (1999). Effect of bacterial biofilm on corrosion of galvanically coupled aluminum and stainless steel alloys under conditions simulating wet storage of spent nuclear fuel. Corrosion 55, 924-936.

Zhou, J. Z., and Ning, D. L. (2017). Stochastic community assembly: does it matter in microbial ecology? Microbiol. Mol. Biol. Rev. 81:e00002-17. doi: 10.1128/ MMBR.00002-17

Zhou, J., Liu, W., Deng, Y., Jiang, Y. H., Xue, K., He, Z., et al. (2013). Stochastic assembly leads to alternative communities with distinct functions in a bioreactor microbial community. mBio 4:e00584-12. doi: 10.1128/mBio.00 584-12

Conflict of Interest: The authors declare that the research was conducted in the absence of any commercial or financial relationships that could be construed as a potential conflict of interest.

Publisher's Note: All claims expressed in this article are solely those of the authors and do not necessarily represent those of their affiliated organizations, or those of the publisher, the editors and the reviewers. Any product that may be evaluated in this article, or claim that may be made by its manufacturer, is not guaranteed or endorsed by the publisher.

Copyright (c) 2021 Van Eesbeeck, Props, Mysara, Petit, Rivasseau, Armengaud, Monsieurs, Mahillon and Leys. This is an open-access article distributed under the terms of the Creative Commons Attribution License (CC BY). The use, distribution or reproduction in other forums is permitted, provided the original author(s) and the copyright owner(s) are credited and that the original publication in this journal is cited, in accordance with accepted academic practice. No use, distribution or reproduction is permitted which does not comply with these terms. 